\title{
Quadrifilar Helical Antenna Array for Line-of-Sight Communications in an Ocean Environment
}

\author{
Michael J. Josypenko, John P. Casey*, and Stephen M. Davis \\ Communications, Imaging, and EW Sensors Department \\ Naval Undersea Warfare Center Division, Newport, RI 02841 \\ Email: [josypenkomj, caseyjp, davissm]@npt.nuwc.navy.mil
}

\section{Introduction}

This paper describes the design of a vertically stacked array of quadrifilar helix antennas that is installed on a buoy that floats along the ocean surface for the purpose of supporting both transmit and receive communications along line-of-sight paths. A quadrifilar helix [1] is a suitable element for this application because it (i) is circularly polarized, (ii) has a wide pattern beamwidth, (iii) has a large pattern front-to-back lobe ratio, and (iv) has an input impedance that is insensitive to tilt angle over the entire range of possible buoy orientations. These characteristics enable an array of these antennas to provide hemispherical coverage overhead with sufficient gain and low sensitivity to multipath interference. However, the design of the array feed network presents a unique and formidable challenge.

In order to send and receive high data-rate communications signals from a buoy in the vicinity of a dynamic sea surface, a suitable high-gain antenna must not couple significantly to sea water. Although other antenna designs such as Counselman's turnstile array [2] work well in a multipath environment when oriented vertically, they suffer significantly from multipath when tilted from the vertical position. Additional requirements for this application include (i) a center frequency of $2.45 \mathrm{GHz}$, (ii) righthand circular polarization (RHCP), (iii) a maximum array length of 18 in., and (iv) the antenna input impedance must be insensitive to buoy tilt up to approximately $20^{\circ}$ from the vertical.

The quadrifilar helix was found to be a suitable antenna element for this application. A parametric study was performed through use of the Numerical Electromagnetics Code (NEC) [3] to determine the optimum helix parameters. The pattern front-to-back lobe ratio was the primary figure of merit used to determine the optimum antenna design. Acceptable front-to-back lobe ratios of $12-17 \mathrm{~dB}$ were found for antennas with pitch angles between $30^{\circ}$ and $50^{\circ}$, element lengths of $1.25-1.5 \lambda$, and radii of approximately $0.125 \lambda$. In order to eliminate the occurrence of grating lobes in the array radiation pattern and allow for a sufficiently long antenna element (for impedance matching), the element separation was set to $0.75 \lambda$, equivalent to 3.61 in. at $2.45 \mathrm{GHz}$. In addition, sufficient spacing between elements was included in order to reduce the interelement mutual coupling. These constraints resulted in the selection of a four-element array of quadrifilar helix elements with pitch angle $=30^{\circ}$, total element length $=1.25 \lambda$, axial length $=0.562 \lambda$, and radius $=0.127 \lambda$.

The original intent was to design the antenna elements to operate above the "cut-in" frequency (i.e., the frequency above which the voltage standing-wave ratio or VSWR becomes small and flat), where the input impedance becomes a broadband resistance. 
However, for the element design length of $1.25 \lambda$, each quadrifilar helix operated at just below the cut-in frequency with an input impedance of $50 \mathrm{ohms}$. In order to adjust the input impedance of each element to the desired value of $100 \mathrm{ohms}$, the conductor width was decreased to one-half of the available space.

\section{Array Design and Assembly}

Fig. 1(a) shows a vertical array consisting of four quadrifilar helices, with each element separated by $0.75 \lambda$ at $2.45 \mathrm{GHz}$. Fig. 1(b) illustrates the feed network. A tube passes through the center of each helix to allow for passage of the feed cables. The tube extends a distance beyond the bottom and top helical elements in order that these elements have the same electrical geometry as the interior elements. For each antenna, a cable penetrates through the cylinder so that the center conductor can feed the element. In the original design, the tube and antenna array were a continuous single piece. However, this was determined to be difficult to assemble, especially in the attempt to pull the feed cables through the tube and slide the array of quadrifilar helices over the protruding center conductors of the four feed points. Consequently, the array was split into four pieces that could be assembled together.

The array assembly (Fig. 2) includes the antenna elements and feed cables along with four support structures, one bottom extension, and one top extension. The tube at the center of the array allows passage of the feed cables. The tube is made of brass, not copper, in order to allow localized heating for soldering. It is stepped at its ends, so that the next antenna element or an extension of the tube can be fitted onto it for continuity with the remainder of the array. At the stepped sections, there are four radial solder holes to allow the solder to flow into the junction between adjacent tube sections and to allow mechanical and electrical bonding of the adjacent sections. There are four radial and circumferentially symmetric spaced holes placed about the tube to allow four feed cables to connect to the four quadrifilar helical elements. The hole was made slightly larger than the 0.040 in. diameter cables to permit soldering the cable to the hole in order to avoid any RF loops that may form. A brass radial ring, soldered to the tube, is used for the feed point for the four elements by allowing the cable outer conductors to be soldered to the radial end of the ring. Ideally, to keep the cable at the same RF potential as that on the ring and tube, the entire length of the cable outside of the hole should be soldered to the tube and ring. In practice, the cable was soldered only to its hole and the end of the ring. The small open loop that results in this incomplete soldering is small enough electrically so that it does not create any RF problems for the antenna. Hard plastic spacers are glued in place on the tube and on the top of the ring to hold the antenna in place and to keep the helical radius constant. A large spacer supports the top of one helix. Fig. 3 gives a close-up view of a quadrifilar helix element that shows the feeds and spacers.

\section{Measured Results}

Fig. 4(a) shows plots of the measured VSWR of both feed arms of a quadrifilar helix element with the brass feed tube. In order to obtain an optimum impedance match at 2.45 $\mathrm{GHz}$, the axial length of the antenna was increased by $0.19 \mathrm{in}$. from the theoretical design value to $2.90 \mathrm{in}$. The plots show that the antenna has a good impedance match (VSWR = 1.3 ) at $2.45 \mathrm{GHz}$. Fig. 4(b) shows measured radiation patterns in the vertical plane of a quadrifilar helix element with the brass feed tube. The patterns show that the antenna produces a maximum radiation overhead with predominantly RHCP in the upper half 
space and left-hand circular polarization (LHCP) over most of the lower half space. Although the radiation in the lower half space is primarily LHCP, the gain in this region is significantly lower than the RHCP gain produced in the upper half space. Consequently, this quadrifilar helix antenna is less sensitive to multipath interference. The front-to-back lobe pattern ratio for this antenna is approximately $11 \mathrm{~dB}$. Although the additional section of brass tube produced a slight change in the measured impedance of the quadrifilar helix, there was no noticeable effect on the radiation pattern.

Future work includes the measurement of the interelement mutual coupling followed by at-sea measurements with the array installed on the buoy

\section{References}

[1] C. C. Kilgus, "Resonant Quadrifilar Helix Design," Microwave Journal, vol. 13, pp. 49-54, December 1970.

[2] C. C. Counselman, "Multipath-Rejecting GPS Antennas," Proceedings of the IEEE, vol. 87, no. 1, pp. 86-91, January 1999.

[3] G. J. Burke, "Numerical Electromagnetics Code - NEC-4.1 - Method of Moments, Parts I and II, UCRL-MA-109338, Lawrence Livermore National Laboratory, Livermore, CA, January 1992.

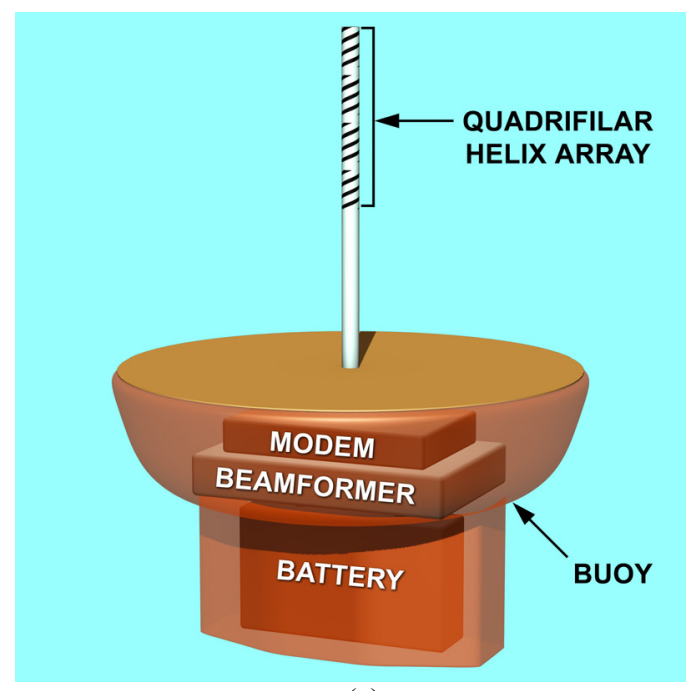

(a)

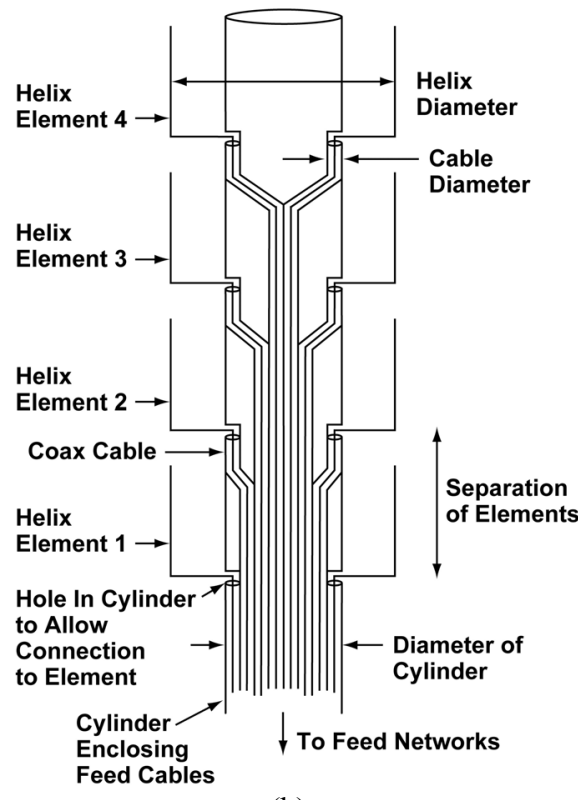

(b)

Figure 1. (a) Four-Element Linear Array of Quadrifilar Helices Installed on a Buoy

(b) Feed Network Through One Bifilar Pair of Quadrifilar Helix Antenna Stack 


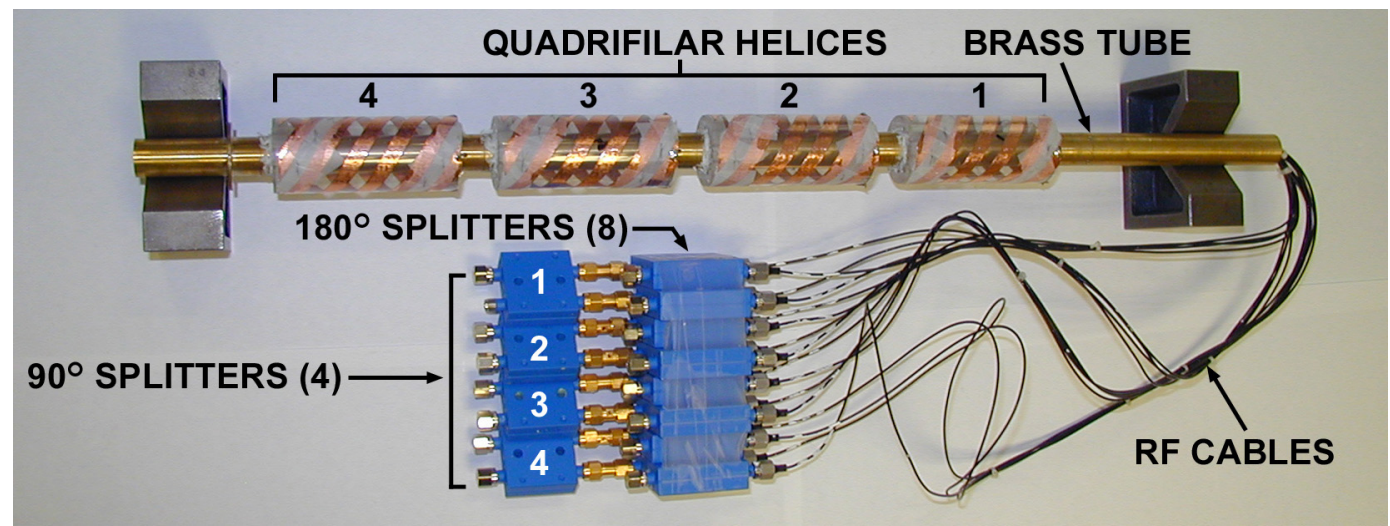

Figure 2. Quadrifilar Helix Array with Feed Assembly

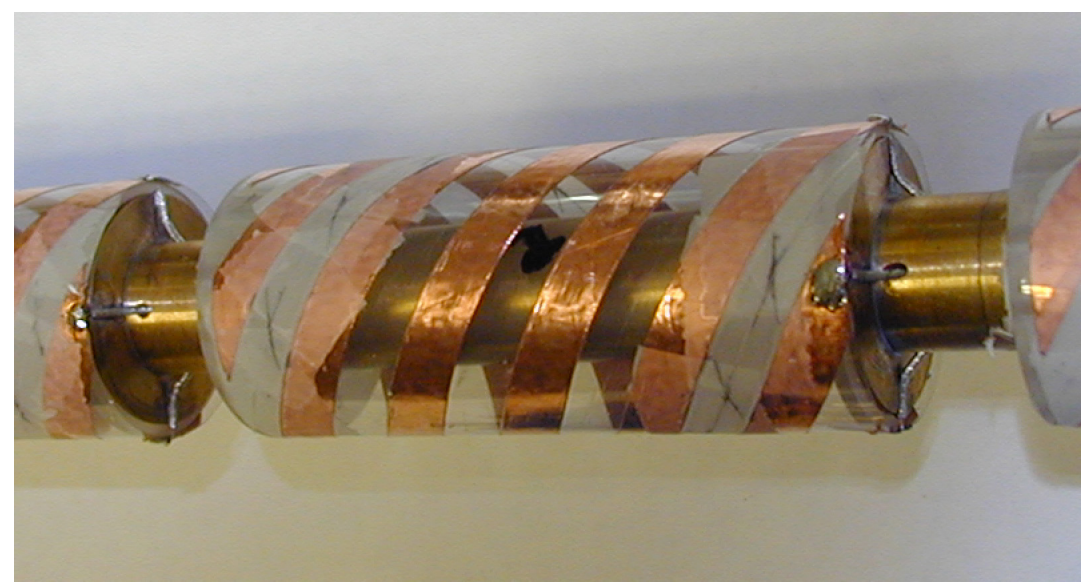

Figure 3. Close-up View of Quadrifilar Helix Element Showing Feeds and Spacers

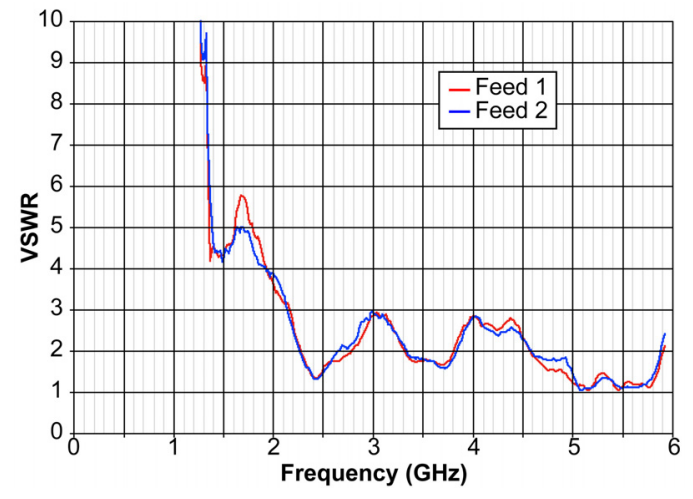

(a)

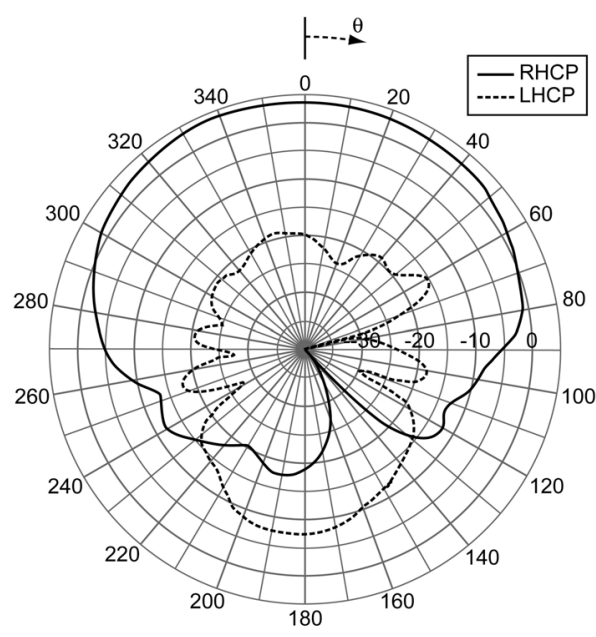

(b)

Figure 4. Measurements of a Quadrifilar Helix Antenna Element with Brass Feed Tube; (a) Input VSWR of Both Feed Arms; (b) Free-Space Radiation Patterns in dB 